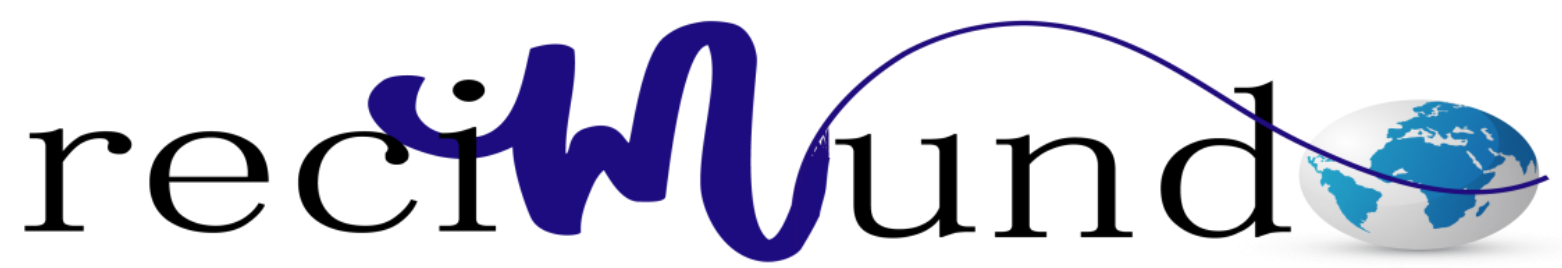

Revista Cientifica Mundo de la Investigación y el Conocímiento

César Bravo Bermeo ${ }^{\text {a }}$; Carmen Lazo Caicedo ${ }^{\text {b }}$; Sonia Cedeño Loor ${ }^{\mathrm{c}}$; Eduardo Vera Muthre ${ }^{\mathrm{d}}$

Uso de morfina en niños

Use of morphine in children

Revista Científica Mundo de la Investigación y el Conocimiento. Vol. 2 núm.3, julio, ISSN: 2588-073X, 2018, pp. 73-94

DOI: 10.26820/recimundo/2.(3).julio.2018.73-94

Editorial Saberes del Conocimiento

Recibido: 05/04/2018

Aceptado: 01/06/2018

Publicado: 30/07/2018

Correspondencia: cesar.bravob@ug.edu.ec

a. Docente Carrera de Medicina. Universidad de Guayaquil; cesar.bravob@ug.edu.ec

b.Docente Carrera de Medicina. Universidad de Guayaquil; carmen.lazoc@ug.edu.ec

c. Docente Carrera de Medicina. Universidad de Guayaquil; leylla.cedenol@ug.edu.ec

d.Docente Carrera de Medicina. Universidad de Guayaquil; eduardo.veram@ug.edu.ec 


\section{Uso de morfina en niños}

Vol. 2, núm. 3., (2018)

César Bravo Bermeo; Carmen Lazo Caicedo; Sonia Cedeño Loor; Eduardo Vera Muthre

\section{RESUMEN}

Se realizó una investigación sobre el uso de morfina en niños mediante una encuesta aleatoria a cincuenta médicos residentes en cinco hospitales con atención pediátrica. La encuesta estaba destinada a establecer la experiencia, la regularidad de la administración y las principales restricciones de su aplicación. Los resultados muestran que el desconocimiento médico y la falta de disponibilidad en las farmacias constituyen las barreras primarias para tratar el dolor severo en la población pediátrica, la situación que contradice la práctica habitual que se lleva a cabo en otras latitudes.

Palabras claves: Morfina, niños, pediatría. 


\title{
Uso de morfina en niños
}

Vol. 2, núm. 3., (2018)

César Bravo Bermeo; Carmen Lazo Caicedo; Sonia Cedeño Loor; Eduardo Vera Muthre

\begin{abstract}
An invstigation was made about morphine use in children troughout an aleatory survey to fifty resident doctors in five hospitals with pediatric attention. The survey was focussed in establishing the experience, regularity of administration and principal restrictions for its application. Results show that the resident doctors unknow and its non availability in drug strores are main obstacles for treating severe pain in pediatric population; situation that contradicts a common practice in other places.
\end{abstract}

Keywords: Morphine, children, pediatrics. 


\section{Uso de morfina en niños}

Vol. 2, núm. 3., (2018)

César Bravo Bermeo; Carmen Lazo Caicedo; Sonia Cedeño Loor; Eduardo Vera Muthre

\section{Introducción.}

El tratamiento adecuado del dolor en los niños responde a la puesta en práctica de variadas consideraciones siendo la primera el establecimiento del grado de afectación de acuerdo con la categorización de: a) leve b) moderado c) severo; y la elección del fármaco apropiado siguiendo los lineamientos diseñados por la OMS en su escala analgésica.

Es necesario, además conocer la fisiopatología del dolor de las enfermedades causales y el mecanismo de acción de los fármacos escogidos, sus contraindicaciones y efectos colaterales. En el caso de dolor severo por cáncer y por enfermedades no oncológicas cuyo dolor la experiencia clínica ha demostrado tiene elevados niveles de control con los fármacos opioides, son los que deben prescribirse. De ellos, la morfina es el fármaco tradicional por su centenaria aplicación, el control efectivo del dolor, su bajo costo, la ausencia de dosis techo, la seguridad en su administración, los efectos colaterales fácilmente controlables.

Sin embargo, la morfina clasificada como analgésico narcótico por su acción euforizante, sedante y la posibilidad de producir adicción junto a la depresión respiratoria por sobredosis han contribuido a que su utilidad en el campo de la medicina no sea aprovechada. Sometida al vaivén de necesidades circunstanciales como analgésico de combatientes, reservado para adultos en fase terminal de enfermedades oncológicas experimenta un acertado y favorable viraje al tratamiento del dolor del niño con enfermedad oncológica y no exclusivamente para la fase terminal.

El compasivo interés por aliviar el dolo de los niños en oposición a las erróneas ideas de que los niños no experimentan dolor o sufren menos que los adultos por su causa, ha conducido a la elaboración de métodos de evaluación del dolor que permiten visualizar su real afectación, a la 


\section{Uso de morfina en niños}

Vol. 2, núm. 3., (2018)

César Bravo Bermeo; Carmen Lazo Caicedo; Sonia Cedeño Loor; Eduardo Vera Muthre investigación tanto experimental como clínica de las acciones y contraindicaciones de la morfina, - referente de los analgésicos narcóticos- a descartar mitos sobre sus propiedades y a eliminar barreras legales que limitan su uso, constituyéndose en el fundamento para la mayor seguridad de prescribirla no solo en el niño con cáncer sino también en el niño con el dolor no oncológico.

\section{Objetivos.}

Considerando la frecuencia con que se atienden pacientes pediátricos con dolor severo de variadas etiologías en los servicios hospitalarios, particularmente en sus áreas de emergencia, y con la percepción que entre los recursos farmacológicos para su tratamiento a la morfina no se le da una prioritaria consideración, nos propusimos demostrarla mediante una encuesta a los residentes de pediatría en el uso de este opioide y confrontar sus resultados con la investigación y experiencia clínica actual sobre su administración a niños en diferentes hospitales del planeta.

\section{Material y Métodos}

En el curso de un mes, año 2009, se realizó un análisis descriptivo mediante una encuesta a 50 médicos residentes de los hospitales pediátricos o con áreas de atención pediátrica de la ciudad de Guayaquil, entre los que se incluyó, los siguientes: Roberto Gilbert Elizalde, Abel Gilbert Pontón, Icaza Bustamante, León Becerra y Naval. Participaron como encuestadores estudiantes de sexto año de medicina, grupo 16 de la Facultad de CC. MM del ciclo de Pediatría del Hospital Abel Gilbert Pontón. Se escogieron 10 residentes de manera aleatoria por cada hospital, no se consideró edad, sexo, nivel de formación, años de experiencia o posición jerárquica. Debían responder al siguiente cuestionario de preguntas abiertas sin limitantes en la 


\section{Uso de morfina en niños}

Vol. 2, núm. 3., (2018)

César Bravo Bermeo; Carmen Lazo Caicedo; Sonia Cedeño Loor; Eduardo Vera Muthre

argumentación, pero, para efectos de hacer objetivas las respuestas se consignaron con un SI o NO.

\section{CUESTIONARIO:}

1. ¿Se puede utilizar morfina en niños?

2. ¿Hay razones válidas para limitar el uso de morfina en niños?

3. ¿Considera que la morfina debe estar disponible en la farmacia de los hospitales pediátricos?

4. ¿Tiene experiencia en el uso de morfina en niños?

5. ¿Únicamente los niños con dolor por cáncer deberían recibir morfina?

6. ¿Piensa que administrar morfina en niños es peligroso?

7. ¿Se debe administrar morfina a niños? Indicaciones.

\section{Respuestas}

TABLA 1

\begin{tabular}{|l|l|l|l|}
\hline Preguntas & & SI & NO \\
\hline $\mathbf{1}$ & Se puede utilizar & $\mathbf{3 5}$ & $\mathbf{1 5}$ \\
\hline $\mathbf{2}$ & Hay razones válidas para limitar su uso & $\mathbf{4 0}$ & $\mathbf{1 0}$ \\
\hline $\mathbf{3}$ & Debe estar disponible en las farmacias de los hospitales & $\mathbf{2 8}$ & $\mathbf{2 2}$ \\
\hline $\mathbf{4}$ & Tienen experiencia & $\mathbf{6}$ & $\mathbf{4 4}$ \\
\hline $\mathbf{5}$ & Únicamente en dolor por cáncer & $\mathbf{2 6}$ & $\mathbf{2 4}$ \\
\hline $\mathbf{6}$ & Es peligroso su uso en niños & $\mathbf{4 5}$ & $\mathbf{5}$ \\
\hline $\mathbf{7}$ & Se debe administrar morfina a niños. Indicaciones & $\mathbf{1 1}$ & $\mathbf{3 9}$ \\
\hline & & $\mathbf{2 0 8}$ & $\mathbf{1 4 2}$ \\
& & & \\
\hline
\end{tabular}




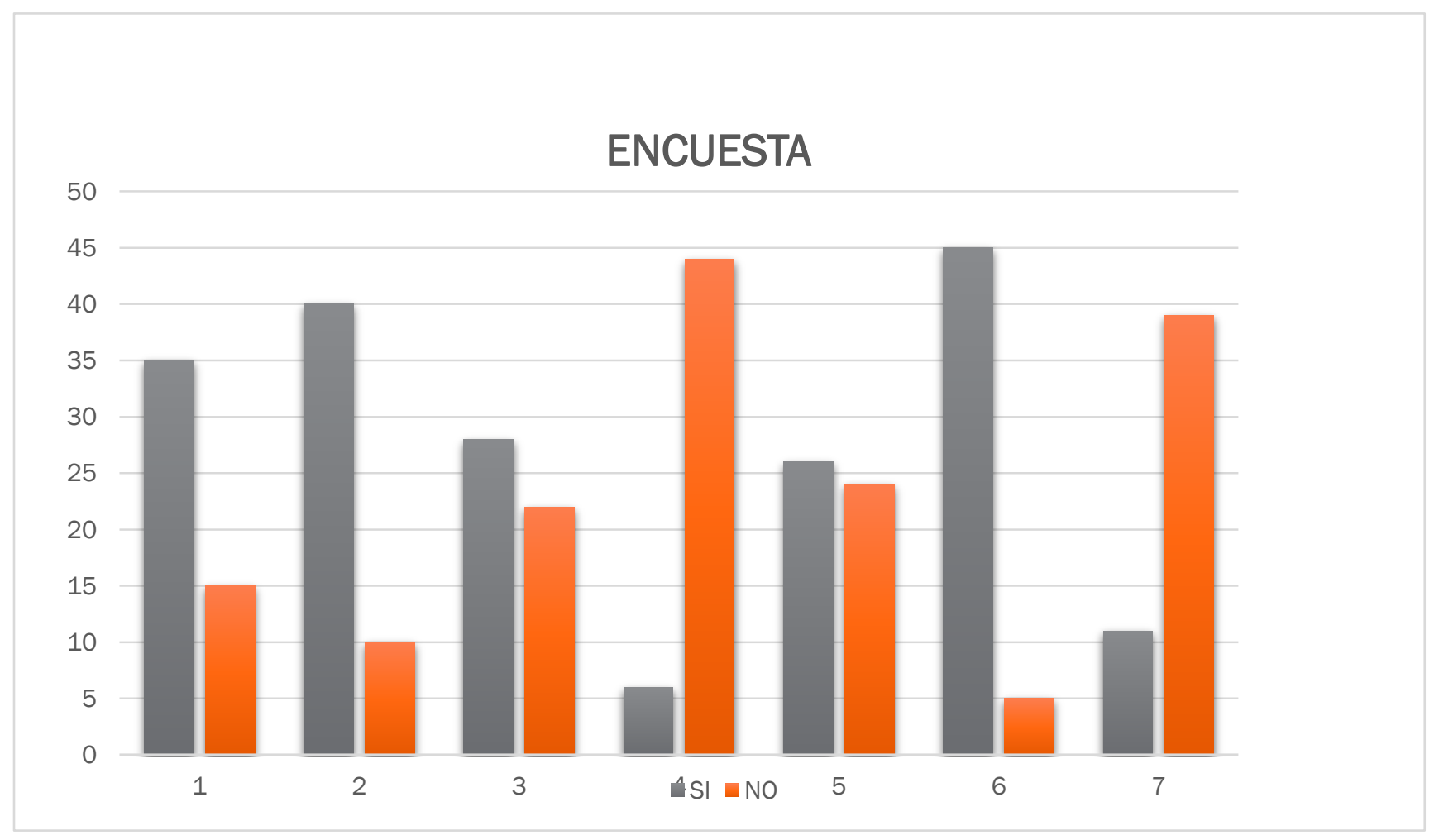

\section{GRAFICO 1.}

\section{Resultados}

En el análisis de los resultados se observaron algunas respuestas contradictorias, ejemplo: tener experiencia y considerar que no se debe administrar morfina los niños; otras que traducen desconocimiento, como sostener que se debe administrar en dolor no oncológico y no mencionar una situación específica, relativizar la respuesta con frases como "dependiendo del caso", relacionar el riesgo con la cautela que se debe tener con la administración de cualquier fármaco, o respuestas muy generales: se debe administrar "si es necesario", en los "dolores intratables, “" en casos extremos", "en casos especiales", etc. 


\section{Uso de morfina en niños}

Vol. 2, núm. 3., (2018)

César Bravo Bermeo; Carmen Lazo Caicedo; Sonia Cedeño Loor; Eduardo Vera Muthre

El 70\% de los encuestados respondió que la morfina se puede administrar a niños; $80 \%$ consideraron que existen razones para limitar su uso; un 56\% que debe estar disponible en las farmacias de los hospitales pediátricos; únicamente $12 \%$ asevero tener experiencia; reservado para el tratamiento del dolor por cáncer 52\%; lo consideran delicado o incluso peligroso un 90\%; se debe administrar para el dolor no oncológico mencionando por lo menos una indicación $22 \%$.

El primero de los porcentajes demuestra que la mayoría de los residentes acepta que la morfina se puede administrar a niños, cifra incrementada por el grupo que considera que únicamente se debe administrar en el dolor por cáncer con desconocimiento de su utilidad en el dolor no oncológico. Señalan también la depresión respiratoria como el factor limitante más importante, efecto colateral que eleva la morfina a la categoría de sustancia peligrosa, destacando que la adicción nunca fue mencionada. Las opiniones divididas en cuanto al aprovisionamiento de morfina en las farmacias son reveladoras de la inseguridad o fobia que se tiene a este fármaco.

\section{Discusión}

Tradicionalmente los médicos han dedicado si atención al diagnóstico de la condición causante del dolor antes que al tratamiento y alivio del mismo. La nueva corriente médica da prioridad al tratamiento del dolor independientemente de los afanes diagnósticos que se realicen. El dolor leve en pacientes pediátricos atendidos de forma ambulatoria en su gran mayoría se resuelve con paracetamol o ibuprofeno; el empleo de otros analgésicos o antiinflamatorios del primer escalón de la escala analgésica enfrentan determinadas limitaciones.

Los opioides débiles indicados en el segundo escalón para el dolor moderado de la escala analgésica tienen muy poca o ninguna aplicación; la morfina encasillada en el tercer escalón para 


\section{Uso de morfina en niños}

Vol. 2, núm. 3., (2018)

César Bravo Bermeo; Carmen Lazo Caicedo; Sonia Cedeño Loor; Eduardo Vera Muthre

tratar el dolor severo, pese a ser el opioide del que más información se tiene y patrón por el que se miden las propiedades analgésicas de otros fármacos, en nuestro medio hospitalario de niños, se observa que su administración casi nunca o nunca se da, como si ocurre en hospitales alrededor del mundo en los que se trata el dolor derivado de condiciones patológicas no oncológicas.

Hay creciente evidencia que demuestra que el dolor no tratado está asociado con adversas consecuencias con compromiso clínico y del desarrollo, pero que estas consecuencias adversas pueden ser prevenidas o atenuadas mediante una apropiada terapia analgésica. Por lo tanto, el tratamiento efectivo del dolor agudo debe ser una prioridad para los niños de todas las edades. Durante las últimas décadas la investigación explorando la evaluación del dolor; el desarrollo de la farmacología de analgésicos, y el uso de analgésicos ha eliminado muchos mitos y errores de concepción acerca del manejo del dolor en pacientes pediátricos; se ha probado que los analgésicos pueden usarse de manera segura en neonatos, lactantes y niños; y proveen una base para el desarrollo de guías del manejo del dolor pediátrico.

Sin embargo, actualmente hay un subdiagnóstico y un subtratamiento del dolor severo. Debido a la opiofobia de numerosos profesionales de la salud muchos pacientes con dolor sensible a los opioides son submedicados, lo que resulta un innecesario aumento de la morbilidad y el sufrimiento. Además, siendo la morfina un fármaco de bajo costo, no tiene un patrocinante interesado en invertir dinero para su promoción en la comunidad médica.

Los adelantos en el manejo del dolor por cáncer con opioide, promovido por un pequeño grupo de especialistas en los 80s, han sido extrapolados a todos los pacientes con dolor crónico. Ahora sabemos que no hay dosis máxima limitante, y muchos pacientes con dolor crónico están 


\section{Uso de morfina en niños}

Vol. 2, núm. 3., (2018)

César Bravo Bermeo; Carmen Lazo Caicedo; Sonia Cedeño Loor; Eduardo Vera Muthre

recibiendo opiáceos de manera prolongada; la ruta intratecal es común para el dolor agudo y crónico; el tratamiento prolongado con morfina y otros opiáceos en algunos lugares ha reemplazado a otros fármacos en el tratamiento del dolor y la restauración funcional.

La Organización Mundial de la Salud (OMS) ${ }^{1}$ elaboro una escalera analgésica que permite un enfoque simple y racional para el alivio del dolor por el cáncer. Los analgésicos simples como el paracetamol se ubican el primer peldaño (dolor leve), los opioides débilescodeína- en el segundo (dolor moderado) y los opioides fuertes- por ejemplo, morfina- en el tercero (dolor intenso). Este enfoque debe tenerse en cuenta junto con otros principios. Primero, en cada etapa también debe considerarse la terapia adyuvante- como radiación- para el dolor óseo metastatico, antiinflamatorios no esteroides para el dolor musculo-esquelético, antidepresivo y anticonvulsivantes para el dolor neuropatico. Segundo, los opioide no deben aplicarse según el dolor sino en forma regular, por reloj. La prescripción de una dosis regular de un opioide fuerte debe acompañarse siempre de una dosis de rescate para el dolor episódico que es una fracción fija de los requerimientos diarios totales. La dosis total debe revisarse cada 48 horas y aumentar la dosis regular si se requirieron más de dos dosis de rescate en periodos consecutivos de 24 horas. Tercero, siempre se prefiere la vía parenteral a menos que exista una contraindicación. 


\section{Uso de morfina en niños}

Vol. 2, núm. 3., (2018)

César Bravo Bermeo; Carmen Lazo Caicedo; Sonia Cedeño Loor; Eduardo Vera Muthre

\section{Grafico 1: OMS Escalera Analgésica}

Medicamentos para

el dolor:

- Vía oral

- Numero de dosis

Usando la escalera

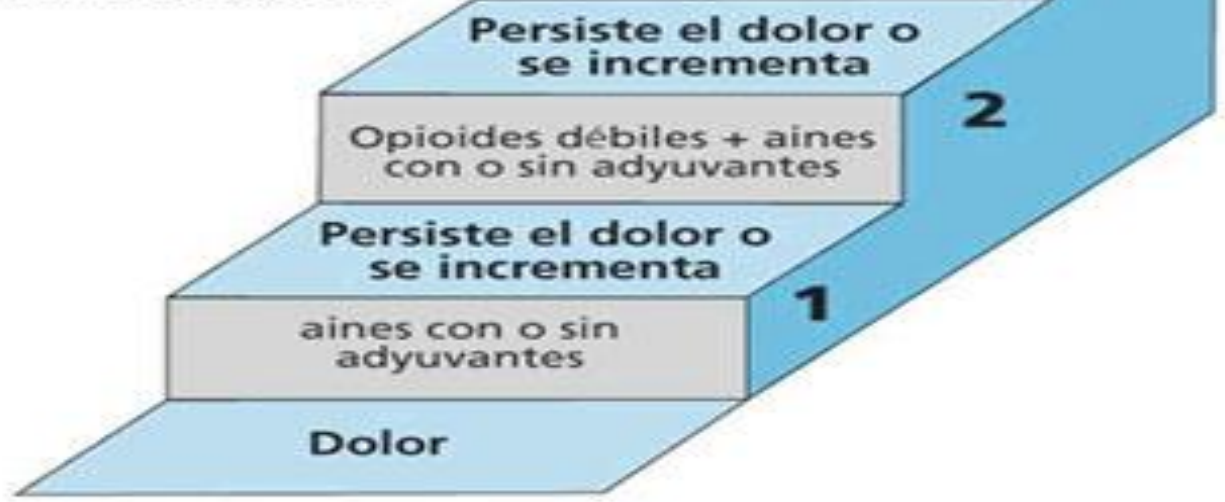

Fuente: wnw.who.int/cancer/palliative/painladder/en

La morfina es el medicamento de elección para controlar el dolor severo en la mayoría de los niños. Tiene una potencia analgésica siete veces mayor que la codeína y diez veces mayor que la meperidina; la duración promedia de su efecto es de 3-4 horas. Puede emplearse por vía oral, subcutánea, intravenosa, epidural e intratecal. La potencia oral comparada con la parenteral es aproximadamente del 50\%, es decir que, para lograr el mismo efecto, la dosis oral de morfina debe ser el doble de la aplicada por vía endovenosa o subcutánea.

Está incluida en la lista Modelo de Medicamentos Esenciales de la $\mathrm{OMS}^{2}$. Aun así, en algunos países se prohíbe su empleo terapéutico y en otro su consumo es restringido. Ello se debe al temor de que su uso prolongado cause adicción y la posibilidad de que se canalice por conductos ilegales. Otro problema es que muchos confunden la adicción (dependencia psicológica) con la dependencia física. 


\section{Uso de morfina en niños}

Vol. 2, núm. 3., (2018)

César Bravo Bermeo; Carmen Lazo Caicedo; Sonia Cedeño Loor; Eduardo Vera Muthre

Esta última es un estado fisiológico en el cual aparecen síntomas y signos de abstinencia cuando se descontinua la administración del opiáceo o se administra un antagonista de este. La dependencia física se manifiesta en casi todos los casos en que ha habido administración continua de un opiáceo y no debe confundirse con adicción.

De las innumerables publicaciones de investigaciones y práctica clínica que se realizan sobre el uso de morfina en niños, hemos seleccionado unas pocas, pero suficientes para incentivar la revisión de su farmacocinética y farmacodinamia como primer paso para que este fármaco a través de su aplicación en los casos seleccionados permita eliminar los motivos preexistentes y lo conviertan en alternativa de primera línea en el tratamiento del dolor severo en niños.

Wilson y Pendleton ${ }^{3}$ concluyeron después de una revisión de 198 expedientes de pacientes con dolor, tales como dolor torácico, dolor abdominal y cólico renal, que la "oligoanalgesia" es prevalente en los departamentos de emergencia. 56\% no recibieron medicación analgésica mientras permanecieron en el departamento de emergencia.

Zoltie y $\mathrm{Cust}^{4}$ fueron los primeros que compararon el uso de opiáceos con placebos en adultos con dolor abdominal agudo. Todos encontraron analgesia segura y efectiva para reducir el dolor sin incremento de la morbilidad. Estos estudios cambiaron la práctica en la emergencia y cirugía de adultos. Ahora esta estandarizado el cuidado de proveer alivio del dolor en adultos con dolor abdominal agudo, antes del diagnóstico e intervención quirúrgica.

En un estudio doble ciego, placebo controlado, con sulfato de morfina y solución salina en adultos mayores de 18 años con dolor abdominal agudo de más de 48 horas de duración se 


\section{Uso de morfina en niños}

Vol. 2, núm. 3., (2018)

César Bravo Bermeo; Carmen Lazo Caicedo; Sonia Cedeño Loor; Eduardo Vera Muthre

observó que efectivamente la morfina alivia el dolor y no altera la habilidad de los doctores para evaluar y tratar adecuadamente a los pacientes ${ }^{5}$.

Green R y colaboradores, demostraron a través de un estudio doble ciego sobre 108 niños en los que 56 recibieron solución salina como placebo y 52 morfina que el fármaco reduce efectivamente la intensidad del dolor abdominal agudo de los niños y parece que no enmascara los signos físicos de apendicitis aguda ${ }^{6}$.

En una revisión sobre los efectos de la administración de opioides sobre el dolor abdominal y el diagnostica acertado en niños con dolor abdominal agudo moderado a severo evaluados en la sala de emergencia, los autores sostienen que la evidencia actual apoya la administración de opioides a niños con dolor abdominal agudo ${ }^{7}$.

Se comparó la eficacia analgésica y la incidencia de efectos colaterales relacionados con la administración de morfina en el postoperatorio de niños en 36 ensayos clínicos. Se concluye que, aunque algunos factores pueden justificar su uso, no tiene un efecto analgésico superior con mayor incidencia de efectos colaterales comparados con los controles ${ }^{8}$.

Un estudio comparativo del uso de analgésicos en pacientes con fractura de extremidades o clavícula entre adultos y niños con documentado dolor moderado o severo establece que la severidad del dolor a menudo no fue registrada y que los pacientes pediátricos reciben menos analgésicos, especialmente narcoticos ${ }^{9}$.

Un estudio prospectivo realizado en la Emergencia Pediátrica del Hospital Necker de Paris que incluía 91 niños entre 5 meses y 6 años de edad con un miembro deformado traumáticamente a los que se les administro morfina oral $(0,5 \mathrm{mg} / \mathrm{kg})$, evidencio la toma del 


\section{Uso de morfina en niños}

Vol. 2, núm. 3., (2018)

César Bravo Bermeo; Carmen Lazo Caicedo; Sonia Cedeño Loor; Eduardo Vera Muthre

medicamento sin dificultades, desaparición del dolor en 30-60 minutos y pocos efectos colaterales. Ninguno severo ${ }^{10}$.

69 pacientes, todos menores de 6 años sometidos a cirugía ortopédica, recibieron morfina intravenosa post operatoria mediante el sistema de analgesia controlada por enfermera siguiendo un protocolo de dosificación. Se valoró la intensidad del dolor y los efectos colaterales. Los resultados evidenciaron seguridad del protocolo, ningún paciente presento depresión respiratoria ni sedación excesiva ${ }^{11}$.

Los analgésicos opioides parentales, son las drogas de elección para tratar las crisis de dolor severo en los niños con enfermedad drepanocitica. Un estudio comprobó la eficacia clínica y seguridad de la morfina oral vs. morfina intravenosa continua en 56 niños entre 5-7 años de edad con enfermedad drepanocitica y episodios de dolor severo. La analgesia opioide se requirió promedio 4.2 días. No hubo diferencias significativas en la frecuencia de rescates entre los grupos oral e IV. Los efectos adversos no difirieron significativamente en frecuencia y severidad. La morfina oral de liberación controlada es una alternativa segura no invasiva a la morfina intravenosa ${ }^{12}$.

Se administró morfina por vía subcutánea después de cirugía mayor a 20 recién nacidos, promedio de edad: 38 5/7. Todos mostraron alivio del dolor sin efectos colaterales severos. La infusión subcutánea de morfina es una alternativa al tratamiento intravenoso ${ }^{13}$.

Una encuesta con siete preguntas consultando 36 centros dirigida a establecer cuantos practican rizotomía dorsal selectiva, la frecuencia de este procedimiento y el manejo del dolor postoperatorio. Con relación al tratamiento del dolor se mostraron los siguientes resultados: 17 


\section{Uso de morfina en niños}

Vol. 2, núm. 3., (2018)

César Bravo Bermeo; Carmen Lazo Caicedo; Sonia Cedeño Loor; Eduardo Vera Muthre

centros administran infusión intravenosa continua de opioide; 7 por vía epidural y 6 por vía intratecal. ${ }^{14}$ en otro estudio se encontró que el uso de infusión continua de morfina y midazolam, junto con ketorolaco es efectivo en el tratamiento del dolor postoperatorio y en el espasmo muscular después de rizotomía dorsal selectiva en 55 pacientes con parálisis cerebral. La mayoría de los pacientes (49) recibieron infusión continua de morfina $20-40 \mathrm{mcg} / \mathrm{Kg}$. No hubo episodios de apnea postoperatoria ni excesiva sedación ${ }^{15}$.

En un estudio de regímenes de analgesia usados en las unidades de quemados en el Reino Unido los opioides intravenosos fue el método más popular para reducir el dolor por quemaduras. La morfina tiene un número de propiedades que contribuyen a su indicación en pacientes con quemaduras y parece ser el más ampliamente usado. Una vez alcanzado un control adecuado del dolor con opioides intravenosos y la ingesta oral sea posible los opioides deberían administrarse por vía oral.

Una encuesta por e-mail sobre la práctica del tratamiento del dolor de niños en centros de quemados del Norte América en la que se preguntaba sobre los métodos de evaluación, y la percepción de la eficacia del efecto analgésico en pacientes hospitalizados y ambulatorios de cuatro (4) grupos de edad, revelo que la morfina intravenosa fue el fármaco más frecuentemente usado para el dolor por quemaduras ${ }^{16}$.

73 niños quemados fueron tratados con una forma galénica de morfina. Se observó supresión del dolor en todos los pacientes sin efectos adversos, por lo que puede ser considerada esta preparación como una opción adecuada para el tratamiento del dolor de pacientes pediátricos debido a sus cualidades analgésicas y enormes ventajas ${ }^{17}$. 


\section{Uso de morfina en niños}

Vol. 2, núm. 3., (2018)

César Bravo Bermeo; Carmen Lazo Caicedo; Sonia Cedeño Loor; Eduardo Vera Muthre

La infusión de morfina de 10-30ug/Kg. /h provee una adecuada analgésica con un aceptable nivel de efectos colaterales cuando se administra con una apropiado monitoreo. La vía subcutánea es una alternativa útil a la vía intravenosa. La Analgesia Controlada por Pacientes (ACP) es ahora usada ampliamente tan tempranamente en niños de 5 años y se compara favorablemente con la infusión continua de morfina en niños mayores. La ACP administrando opioides es aplicable después de procedimientos de cirugía mayor, en anemia drepanocitica, en manejo del dolor debido a mucositis, y en el tratamiento de niños con dolor crónico. Esta tecnología puede ser usada también en neonatos donde una dosis bolo sin una infusión previa permite a la enfermera titular administrar al niño para analgesia o para anticipar los episodios de dolor aprovechando el efecto prolongado debido a la lenta eliminación de morfina ${ }^{18}$.

La depresión respiratoria y la adicción, junto al empleo de la morfina en "situación de enfermedad terminal", constituyen los llamados "mitos de la morfina", creencias que se encuentran arraigadas tanto a nivel popular como profesional. El proceso de entrenamiento medico tiende a estimular actitudes negativas hacia el uso de fármacos opioide. No debe temerse al uso de opioide en Pediatría, dado que los riesgos de toxicidad grave o adicción son muy bajos cuando se utilizan en forma terapéutica y racional

Por otro lado, la dependencia psicológica o adicción ha sido definida por la OMS como un estado psíquico y a veces físico, que resulta de la interacción entre un ser viviente y una droga, y se caracteriza por respuestas comportamentales que siempre incluyen la compulsión para conseguir la droga de forma continua o periódica, con el propósito de experimentar sus efectos psíquicos. 


\section{Uso de morfina en niños}

Vol. 2, núm. 3., (2018)

César Bravo Bermeo; Carmen Lazo Caicedo; Sonia Cedeño Loor; Eduardo Vera Muthre

Varios estudios demuestran que la adicción ocurre muy raras veces cuando la morfina se utiliza solamente para evitar el dolor y que la desviación de la droga hacia mercados ilegales es muy baja en función del volumen mundial de transacciones que se realizan anualmente. Los estudios también concluyen que un incremento en el uso de la morfina no necesariamente implica que se haya incrementado su desviación de los mercados legales a los ilegales.

Con el propósito de controlar el mercado ilegal, muchos países han adoptado regulaciones y legislación que restringen el uso médico y científico de los medicamentos opiáceos, lo que redunda en un deterioro de la calidad de vida de los pacientes. Para poner en ejecución un programa de alivio de dolor en el cáncer, la OMS recomienda que los gobiernos de los Estados Miembros acojan las medidas específicas en cuanto a políticas gubernamentales, educación y disponibilidad de opiáceos.

Human Rights Watch ${ }^{19}$ exhorta a todos los países a desarrollar e implementar políticas sobre cuidados paliativos y tratamiento del dolor, si es que aún no lo han hecho; a revisar sus regulaciones de narcóticos a fin de asegurar que no interfieran en el uso médico de la morfina y otros medicamentos opiáceos, así como a garantizar que el tratamiento paliativo y del dolor se incluya en los currículos de capacitación para el personal médico y de enfermería.

Para la Jefatura Internacional de Fiscalización de Estupefacientes (JIFE) existen varios factores que han limitado en países en desarrollo como el Ecuador una disponibilidad equitativa y una buena relación costo eficacia de drogas para el alivio del dolor, tales como la falta de recursos financieros, baja prioridad de la autoridad gubernamental, así como los temores infundados y los prejuicios arraigados en cuanto al uso de opiáceos con fines medicos ${ }^{20}$. 


\section{Uso de morfina en niños}

Vol. 2, núm. 3., (2018)

César Bravo Bermeo; Carmen Lazo Caicedo; Sonia Cedeño Loor; Eduardo Vera Muthre

Los análisis efectuados por la JIFE y la $\mathrm{OMS}^{20}$ han relevado que las dificultades para garantizar el acceso a los analgésicos opioides radican en diversos factores relacionados, entre ellos una formación inadecuada de los profesionales de la salud y la falta de conocimientos y aptitudes en la materia de tratamiento del dolor, la actitud de la opinión pública, impedimentos reglamentarios y limitaciones económicas. La JIFE ha pedido a los gobiernos que determinen los impedimentos que existan en sus países y adopten medidas para aumentar la disponibilidad de estos medicamentos.

\section{Conclusión.}

Estos resultados revelan que los residentes de Pediatría están pocos familiarizados con el tratamiento adecuado del dolor severo, esto se origina en la poca o casi nula información que sobre el dolor y su tratamiento se imparte tanto a nivel de las áreas básicas como en las clínicas de la formación médica y se continúa esta situación inalterable en el postgrado.

En esta investigación se pudo apreciar que la mitad de los encuestados consideran que la morfina está reservada para el dolor del paciente oncológico; la conjugación de falta de disponibilidad y el temor a la depresión respiratoria, ni siquiera permite pensar en morfina como alternativa válida para el dolor y si bien un porcentaje considerable de los encuestados sabe que la morfina se puede utilizar en el dolor no oncológico, no tienen experiencia, por lo tanto, jamás se atreverían a usar morfina en un niño con dolor severo no oncológico.

Es la catedra de Farmacología donde se debería iniciar a los estudiantes tanto en los aspectos teóricos y de experimentación sobre hipoanalgesicos y la emblemática morfina para continuar con su aplicación y observación de las respuestas de los enfermos en los centros 


\section{Uso de morfina en niños}

Vol. 2, núm. 3., (2018)

César Bravo Bermeo; Carmen Lazo Caicedo; Sonia Cedeño Loor; Eduardo Vera Muthre hospitalarios de adultos y niños. Únicamente con la experiencia derivada del uso de manera regular en pacientes oncológicos y no oncológicos se podrán eliminar los mitos que pesan sobre esta droga. También debieran revisarse las políticas gubernamentales que limitan la disponibilidad de la morfina en los hospitales y las restricciones a su prescripción.

Es inexplicable que, con los conocimientos profundos alcanzados sobre la génesis del dolor, la atención dada a su control, el establecimiento de clínicas destinadas al tratamiento exclusivo del dolor y la eficacia demostrada de la morfina tanto en niños como en adultos, oncológicos o no, en nuestro medio esta sustancia continúe mantenida en el oscurantismo de la ignorancia y el temor derivado de aquella.

Por otra parte, es alentador que, al momento en nuestro país, el Instituto Nacional de Higiene "Leopoldo Izquierda Pérez" de Guayaquil luego de calificarse en el Consejo Nacional de Estupefacientes (CONSEP) importa el clorhidrato de morfina y compra los servicios de una empresa farmacéutica privada para la producción de ampollas de morfina de 10 y $20 \mathrm{mg}$ que luego son distribuidas y vendidas a nivel nacional.

Con este antecedente y el principio activo disponible se ha propuesto la elaboración de solución oral de morfina por parte del Ministerio de Salud a través de INH como alternativa viable y de bajo costo que mejoraría la disponibilidad y acceso al opioide. 


\section{Uso de morfina en niños}

Vol. 2, núm. 3., (2018)

César Bravo Bermeo; Carmen Lazo Caicedo; Sonia Cedeño Loor; Eduardo Vera Muthre

COMENTARIO DE UNS RESIDENTE DE SEGUNDO AÑO DEL HOSPITAL GARRAHAN DE BUENOS AIRES, ARGENTINA, CON RELACIÓN AL EMPLEO DE MORFINA EN NIÑOS:

"Puedo decir que en el hospita los residentes utilizamos la morfina principalmente en los pacientes posquirúrgicos de neurocirugía y traumologia, según el grado de dolor que manifieste el paciente, la dosis de morfina se ajusta según el paciente controle o no el dolor. Por otro lado, en pacientes con patologías crónicas que usan morfina como medicación de base en ocasiones ingresan por mal manejo del dolor, lo que hacemos es dar dosis de rescate, es decir, dosis extras y si tenemos que usar dos dosis de rescate o más, inmediatamente subimos la dosis siguiente, el 25\% de la habitual y así vamos titulando según lo requiera el paciente. No tiene dosis máxima. Las dosis de morfina que se utiliza son $0,05 \mathrm{mg} / \mathrm{Kg} /$ dosis por vía $\mathrm{EV}$ y $0.1 \mathrm{mg} / \mathrm{Kg}$ por vía oral” V.B.D. 


\section{Uso de morfina en niños}

Vol. 2, núm. 3., (2018)

César Bravo Bermeo; Carmen Lazo Caicedo; Sonia Cedeño Loor; Eduardo Vera Muthre

\section{Bibliografía}

1. Organización Mundial de la Salud. Cancer Pain Relief. Geneve Organización Mundial de la Salud. Genova. 1986

2. Organización Mundial de la Salud. Uso de medicamentos esenciales: lista modelo de medicamentos escenciales (octava lista), sexto informe del Comité de Expertos de la OMS, Ginebra: OMS; 1995.

3. Wilson JE, Pendelton JM. Am J Emergency Med. 1989; 7(6): 620-3

4. Zoltie N, Cust MP. Analgesia in the acute abdomen. Annals of the Royal College of Surgeons of England 198668.

5. Pace $\mathrm{S}$, Burke TF. Intravenous morphine for early pain relief in patient with acute abdominal pain. Acad Emerg Med 1996;3: 1086-1092

6. Green R, Bulloch B, Kabani AQ, Huancoch B, Tenenbein M.A. Early analgesia for children with acute abdominal pain. Pediatrics 2005; 116, 978-983

7. Klein-Kremer A, Goldman RD. Opiod administration for acute abdominal pain in the pedriatric emergency department. J Opioid Manag. 2007; 3(1): 11-4

8. Duedal TH, Hansen EH. A quilitative systematic review of morphine treatment with potoperative pain. Pediatric Anaesth 2007; 17(8):756-74.

9. Brown JC, Klein EJ, Lewis CW, Johnston BD, Cummings P. Emergency Department analgesia for fracture pain. Ann Emerg Med 2003; 42(2): 197-205.

10. Wille C, Bocquet N, Cojocuru B, Leis A, Cheron G, Oral morphine administration for children's traumatic pain. Arch Pediatr 2005; 12(3)248-53

11. Garcia-Gomez $\mathrm{M}$ et al. Treatment of postoperative pain with morphine hydrochloride in children under six years of age in general hospital wards. Orthopaedic Proceedings. 2018. 86-B(SUPP II):141.

12. Jacobson SJ, Koprecky EA, Joshi P, Babul N.Randomized trail of oral morphine for painful episodes of sickle cell disease in children. Lancet 1997; 350:1358-1361

13. Rouss K, Gerber A, Abisetti M, Berner V. Long term subcutaneous morphine administration after surgery in newborns. J Perinat Med.2007; 35(1), pages 79-81

14. Hesselgard K, Reinstrup P, Strumblad L, Unden J, Rommer B. Selective dorsal rhizotomy and postoperative pain management. Pediatric Neurosurgery 2007; 43: 107-112

15. Lieh-Hai MW, Kauffman RE, Uy GH, Damjin M, Simpson PM. A randomized comparison of ketoralac tromethamine and morphine for postoperative analgesia in critically ill children. Crit Care Med. 1999, 27: 2786-91

16. Martin-Herz, Patterson DR, Honari S, Gibbon G, Gibran N, Heimbach DM, Pediatric pain control practice of North American Cenrters. J. Burn Care Rehabil. 2003; 24(1): 26-36D 


\section{Uso de morfina en niños}

Vol. 2, núm. 3., (2018)

César Bravo Bermeo; Carmen Lazo Caicedo; Sonia Cedeño Loor; Eduardo Vera Muthre

17. Amirat-Comvalier V, et al. Preparation and use of morphine capsules in pediatric patient with burn. J. Pharm Bel 1977; 52(6):217-8

18. Norton M. Managment of postoperative pain in children. Archives od Disease in Childhood. Education and Practice 2007:92 (ep14):1-19

19. Junta Internacional de Fiscalización de Estupefacientes (JIFE). Informe anual 1999.

20. Junta Internacional de Fiscalización de Estupefacientes (JIFE). Informe anual 2008 